\title{
MAPAS CONCEITUAIS E APRENDIZAGEM SIGNIFICATIVA NO ENSINO DE REDE DE COMPUTADORES DO CURSO SUPERIOR DE TELECOMUNICAÇÕES
}

\author{
Suélly Lima dos Santos, Instituto Federal Fluminense-IFF Campus Campos Centro \\ suelsster@gmail.com \\ Marco Antonio Gomes Teixeira da Silva, IFF Campus Campos Centro \\ marcoagts@gmail.com \\ Suzana da Hora Macedo, IFF Campus Itaperuna \\ shmacedo@iff.edu.br
}

\section{Resumo}

A proposta deste artigo é apresentar a utilização de mapas conceituais como ferramenta para o ensino-aprendizagem em rede de computadores num curso superior de telecomunicações. Esta experiência é baseada na teoria da aprendizagem significativa. A metodologia empregada foi um experimento conduzido em sala de aula onde os alunos puderam produzir os mapas conceituais de acordo com os temas estudados. Este trabalho tem como objetivo proporcionar aos docentes novas situações potencialmente significativas de ensino-aprendizagem da cadeira, utilizando mapas conceituais. Como conclusão levou-se em consideração os comentários e avaliações que foram feitos para o término do trabalho, destacando-se as vantagens no processo de aprendizagem.

Palavras-chave: Aprendizagem significativa, mapas conceituais, redes de computadores.

\section{CONCEPTUAL MAPS AND MEANINGFUL LEARNING IN SCHOOL OF COMPUTER NETWORK TELECOMMUNICATIONS SUPERIOR COURSE}

\begin{abstract}
The purpose of this paper is to present the use of concept maps as a tool for teaching and learning computer networking a degree in telecommunications. This experiment is based on the theory of meaningful learning. The methodology used was an experiment conducted in the classroom where students were able to produce concept maps according to the topics studied. This paper aims to provide teachers new situations potentially significant teaching and learning of the chair, using concept maps. In conclusion took into account the comments and evaluations were made for the completion of the work, highlighting the advantages in the learning process.
\end{abstract}

Key-words: Meaningful learning, concept maps, computer networks.

\section{Introdução}

Com o advento do computador novos espaços de aprendizagem começam a ser planejados e construídos, não mais restritos ao perímetro em que ocorre uma relação tradicional e fechada entre professores e alunos. A informática permite repensar de forma mais dinâmica e com novos enfoques o universo do conhecimento a trabalhar, criando novas formas de aprendizagem e de comunicação, estimulando a participação ativa dos alunos no processo educativo, instigando-os a conhecer o mundo de forma mais crítica, contando com o professor como orientador desse processo. Como afirmam Leite et al, "assim, a construção do 
conhecimento pode e deve ser mediada por recursos mais agradáveis e estimulantes" (Leite et al, 2010, p.11).

Tendo a seu favor a versatilidade de aplicação e a sua adaptabilidade a diversas atividades, a informática pode promover a integração curricular, a quebra de barreiras entre as disciplinas e entre as diversas culturas, enriquecendo a formação dos alunos e contribuindo para elevar o nível cultural e tecnológico dos educandos. Ainda, segundo Leite et al, "as tecnologias digitais firmam-se, de forma crescente, como instrumentos mediadores nos processos de ensino e aprendizagem" (Leite et al, 2010, p.2).

Nesse contexto, a informática pode vir a contribuir positivamente para o aceleramento do desenvolvimento cognitivo e intelectual do aluno, em especial no que esse desenvolvimento diz respeito ao raciocínio lógico e formal, à capacidade de pensar com rigor e sistematicidade, à habilidade de inventar ou encontrar soluções para problemas (Costa, 1998). Pode também possibilitar ao educando o desenvolvimento de sua capacidade de aprender a aprender, estimulando sua autonomia - que tem como fundamento o aprender fazendo - experimentando e criando. Essa é hoje uma prioridade da escola que, utilizando-se dessa nova tecnologia, pode tornar esse processo mais rico e prazeroso (Coll, 2000). Sendo assim, nas palavras de Leite et al, "as TICs (Tecnologias da Informação e Comunicação) permitem a organização de currículos mais flexíveis, adaptados aos estilos individuais de aprendizagem, além de promover maior autonomia acadêmica e profissional." (Leite et al, 2010, p. 3).

A Figura 1 apresenta o mapa conceitual de como os recursos computacionais podem ser utilizados nas atividades pedagógicas.

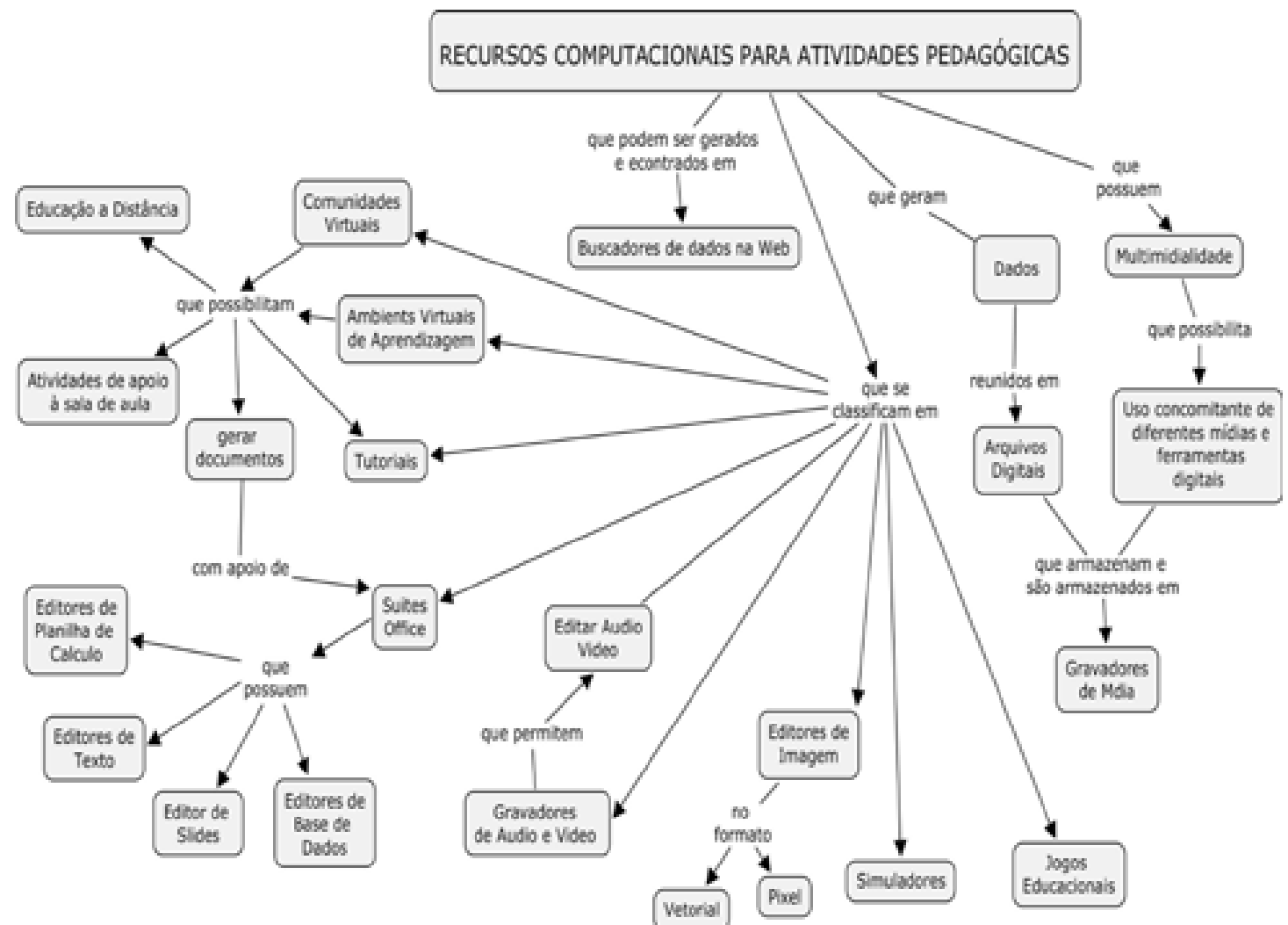

Figura 1 - Como os recursos computacionais podem ser utilizados nas atividades pedagógicas Fonte: Albuquerque et al, 2009

Este trabalho apresenta o uso do mapa conceitual utilizado como ferramenta no processo de ensino-aprendizagem da disciplina redes de computadores em uma turma 
superior tecnológico de telecomunicações. O objetivo é proporcionar aos professores novas situações potencialmente significativas de ensino-aprendizagem da cadeira utilizando mapas conceituais.

\section{Curso superior de tecnologia em telecomunicações}

O curso em estudo foi reconhecido pela Portaria $n^{\circ} 18$ de 2 de janeiro de 2007 está estruturado de modo a buscar desenvolver as competências profissionais que delineiam os traços preponderantes ao perfil do tecnólogo, através da construção do conhecimento técnico associado a uma visão humanística, científica e mercadológicas das telecomunicações (IFF, 2011).

O profissional desta área atua planejando, implantando e operando sistemas de telecomunicações. Processamentos da informação, elementos constituintes das redes de telecomunicações e eletrônica, são fundamentais para o desempenho deste profissional, cujo campo de atuação inclui indústrias, empresas do setor de telefonia fixa e móvel, telemática, televisão aberta e por assinatura, internet, radiodifusão, rádiotransmissão, dentre outros.

Neste trabalho, os mapas conceituais foram utilizados para o enriquecimento do estudo do aluno, possibilitando uma melhor compreensão dos conteúdos estudados.

\section{Tecnologias das Redes}

As redes de dados modernizam, aquecem e molda a sociedade como um todo, além da já tão divulgada economia do mercado capitalista. Diante de tal afirmativa pode ser destacado três fatores que levam a popularização das redes de dados: (i) vertiginosa evolução da eletrônica, que proporciona novos equipamentos, cada vez mais ágil e com menores custos; (ii) o direcionamento da tecnologia para a ciência da informação; e (iii) a rede mundial de computadores, ou seja, a internet (Bolzani, 2004, p. 3).

É inegável que o número de tecnologias disponíveis no mercado proporcionam estudos dos mais diferenciados, sejam quanto aos protocolos, meios físicos de conexão, segurança ou ainda da própria aplicação de cada tecnologia e suas possíveis finalidades. Também é notória que a tecnologia não é estática, logo a forma de passar a informação no ambiente educacional, devem crescer assim como crescem as tecnologias.

A pesquisa na área tecnológica pode ser dita que é infinita, seguir uma linha de raciocínio e um delineador de assuntos são tarefas de professores especialistas na área. Além das questões de conectividade física das tecnologias das redes, cabem ainda, as questões de conectividade lógicas como os protocolos e seus respectivos padrões de conectividade.

\section{Modelo em camadas}

A cadeira rede de computadores do curso superior em sistemas de telecomunicações, assim como a maioria dos cursos superiores na área de tecnologias, tem como base o Modelo em Camadas TCP/IP (Transmission Control Protocol/Internet Protocol - Protocolo de Controle de Transmissão/Protocolo de Internet) e o modelo de referência ISO/OSI (International Organization for Standardization/Open Systems Interconnection - Organização Internacional para Padronização/Interconexão de Sistemas Abertos).

Vale ratificar que entender cada um dos modelos em camadas, não é uma tarefa fácil, definir ainda que os dois modelos realizem as mesmas atividades, porém, com números de camadas diferenciadas é um pouco mais complicado para o indivíduo, que chega a disciplina de redes. Dentro das possíveis dificuldades encontram-se: (i) número de camadas diferentes, 
com atividades resultantes entre elas iguais; (ii) algumas camadas do modelo OSI possuem atividades simplificadas e outras do modelo TCP/IP possuem concentração de muitas informações; (iii) os modelos são apresentados em quatro (TCP/IP) e sete camadas (OSI), no entanto, são estudados em cinco camadas), como é a proposta do livro de Tanenbaum de 1999 e suas edições.

Além das dificuldades acima, que são pautadas por vários professores da disciplina de redes, o indivíduo é inserido em um ambiente totalmente novo dos protocolos e suas finalidades, fica ainda a questão de que o Modelo OSI é mais novo do que o modelo TCP/IP. O qual é citado por Tanenbaum (2003, p. 44) como a "avó" de todas as redes de computadores geograficamente distribuídas, conhecida antes como ARPANET, do Departamento de Defesa dos Estados Unidos. A suíte de protocolos TCP/IP, por sua vez é o protocolo aplicado na Internet e não o modelo OSI.

\section{Aprendizagem Significativa}

Esta proposta pedagógica está baseada na Teoria da Aprendizagem Significativa de David Ausubel. "Aprendizagem significativa é um processo pelo qual uma nova informação se relaciona com um aspecto relevante da estrutura de conhecimento do indivíduo" (Moreira e Masini, 2001).

"Mapas conceituais, ou mapas de conceitos, são apenas diagramas indicando relações entre conceitos, ou entre palavras que usamos para representar conceitos" (Moreira, 1997).

Este trabalho pretende a partir de conceitos já existentes na estrutura cognitiva do aluno do curso de telecomunicações, conceitos tais como sistemas de comutação, de transmissão e radiodifusão, redes de acesso e telemática; sistemas de telecomunicações.

Segundo Moreira (2006, p. 15) “o subsunçor é um conceito, uma idéia, uma proposição já existente na estrutura cognitiva, capaz de servir de 'ancoradouro' a uma nova informação de modo que esta adquira, assim, significado para o indivíduo (isto é, que ele tenha condições de atribuir significados a essa informação)". Nesse aspecto os conceitos previamente "ancorados" na estrutura cognitiva do aluno, tais como arquitetura e técnica de transmissão, redes de acesso, sistemas de comunicação e sistemas e rádio, servirão como subsunçores, para que uma nova informação seja adquirida por parte do educando. Como nova informação, entende-se os conceitos necessários ao ensino de redes de computadores.

De acordo com Moreira (2006, p. 13), a idéia mais importante da teoria de Ausubel pode ser resumida na seguinte proposição do próprio Ausubel “[...] o fator isolado mais importante que influencia a aprendizagem é aquilo que o aprendiz já sabe. Averigue isso e ensine-o de acordo." (Ausubel et al, 1980, p. viii)

Ainda citando Moreira (2006), Ausubel está se referindo à estrutura cognitiva do aprendiz. É preciso que o conteúdo seja aprendido de forma significativa.

Também, quando Ausubel se refere "àquilo que o aprendiz já sabe", para que ocorra a aprendizagem de uma nova informação, o mesmo está se referindo a aspectos específicos da estrutura cognitiva.

Moreira (2006, p. 14), corrobora afirmando que averiguar seria descobrir a estrutura préexistente, os conceitos, que já existem na mente do indivíduo, sua organização e suas interrelações; fazer um "mapeamento" da estrutura cognitiva, o que também é algo difícil de realizar. "Esta informação serve como subsídio para que seja planejada a utilização de mapas conceituais como recurso didático" (Silveira, 2008, p.95).

$\mathrm{Na}$ proposição de Ausubel, "ensine-o de acordo", Moreira (2006) afirma que significa basear o ensino no que o aprendiz já sabe, e identificar isso também não é tarefa fácil. 
Este trabalho propõe, a partir de conceitos já existentes na estrutura cognitiva do aluno do curso superior de telecomunicações, que o mesmo os utilize como subsunçores de forma a alicerçar os novos conceitos a serem apreendidos.

Nesse aspecto os conceitos previamente "ancorados" na estrutura cognitiva do aluno, servirão como subsunçores, para que uma nova informação seja adquirida por parte do educando. Como o ensino de redes de computadores ocorre no último período do curso de sistemas de telecomunicações, o aluno já possui os conceitos de conectividade e tecnologias de conexão bem fundamentada, servindo, portanto, como subsunçores ao novo aprendizado. Ausubel afirma que:

A essência do processo de aprendizagem significativa é que as ideias expressas simbolicamente são relacionadas às informações previamente adquiridas pelo aluno através de uma relação não arbitrária e substantiva (não literal). Uma relação não arbitrária e substantiva significa que as idéias são relacionadas a algum aspecto relevante existente na estrutura cognitiva do aluno (Ausubel et al, 1980, p. 34).

Sendo assim, é importante que o aluno relacione os novos julgamentos com conceitos pré-existentes na sua estrutura cognitiva. Neste caso, tais conceitos são alicerçados nas disciplinas de antecedentes. Ainda, de acordo com Moreira:

[...] uma das condições para ocorrência de aprendizagem significativa é que o material a ser aprendido seja relacionável (ou incorporável) à es trutura cognitiva do aprendiz, de maneira não arbitrária e não literal (Moreira, 2006, p. 19).

Portanto, a Teoria da Aprendizagem Significativa será de grande valia e será usada como fundamentação teórica neste trabalho.

\section{Mapas Conceituais}

Mapas conceituais estão muito ligados à teoria da aprendizagem significativa proposta por Ausubel. Porém, Ausubel nunca falou de mapas conceituais em sua teoria. Esta é uma técnica desenvolvida por Joseph Novak e seus colaboradores na Universidade de Cornell (EUA) (Moreira, 2010, p.17). Portanto, os mapas conceituais foram desenvolvidos por Joseph Novak como ferramenta de característica construtivista para suporte à Aprendizagem Significativa de Ausubel. São diagramas usados para representar, descrever, estruturar, comunicar conceitos e as relações entre eles. Os conceitos constituem os nós do mapa e as relações são os links. Geralmente, os conceitos são substantivos e as relações são representadas por expressões verbais.

Os mapas conceituais podem ser utilizados como instrumento de avaliação da aprendizagem, estratégia de estudo, apresentação de conteúdos, recurso de aprendizagem, entre outros. "Mapas conceituais podem ser utilizados na obtenção de evidências da aprendizagem significativa, ou seja, na avaliação da aprendizagem." (Moreira, 2010, p. 22). Porém é preciso ter cuidado, porque, assim como os mapas conceituais têm significados pessoais, alguns mapas são muito pobres e demonstram que não houve a compreensão do conteúdo desejado. "Os indícios da aprendizagem significativa serão verificados nos registros dos alunos por meio dos relatórios e principalmente, nos mapas conceituais construídos" (Carvalho et al, 2010).

\section{Experimento realizado em sala de aula}


Este experimento foi realizado utilizando a ferramenta CMapTools que é open source e o seu download pode ser feito em: http://cmap.ihmc.us/download/.

Neste estudo participaram discentes que cursava a disciplina redes de computadores do turno noturno do $6^{\circ}$ período do curso tecnológico de telecomunicações do Instituto Federal Fluminense (IFF) campus Campos Centro. Foi considerado que a turma já estava no último período, portanto já possuía conhecimento prévio dos conceitos necessários como subsunçores no experimento. Como afirma Silveira, "o reconhecimento das habilidades prévias é importante na tentativa de identificar subsunçores pré-existentes na estrutura cognitiva dos alunos" (Silveira, 2008, p. 95).

A turma tinha um total de doze alunos com idade entre de 19 a 40 anos, sendo cinco homens e sete mulheres. O experimento foi realizado em dois momentos no segundo semestre de 2010. No primeiro momento, realizou-se a apresentação do mapa conceitual, para que o indivíduo pôde-se conhecer a ferramenta e, no segundo momento iniciou-se a disciplina de redes de computares com o mapa conceitual dos modelos em camadas. As atividades em sala de aula foram desenvolvidas através de aulas expositivas com apostila como material de apoio, sobre os respectivos assuntos a serem trabalhados.

Segundo Moreira (2010, p.16), "é possível traçar-se um mapa conceitual para uma única aula, para uma unidade de estudo, para um curso ou, até mesmo, para um programa educacional completo".

No experimento foi aplicado o estudo dos dois modelos TCP/IP e OSI simultaneamente para facilitar a visualização das funções de cada segmento, refletindo numa aula dinâmica e possibilitando a interação por parte do alunado.

Antes do estudo de cada segmento, retornou-se ao mapa conceitual inicial, apresentado no início da disciplina (Figura 2). Ao final desta etapa de interação com os modelos, as camadas foram "estouradas" (Figura 3). 


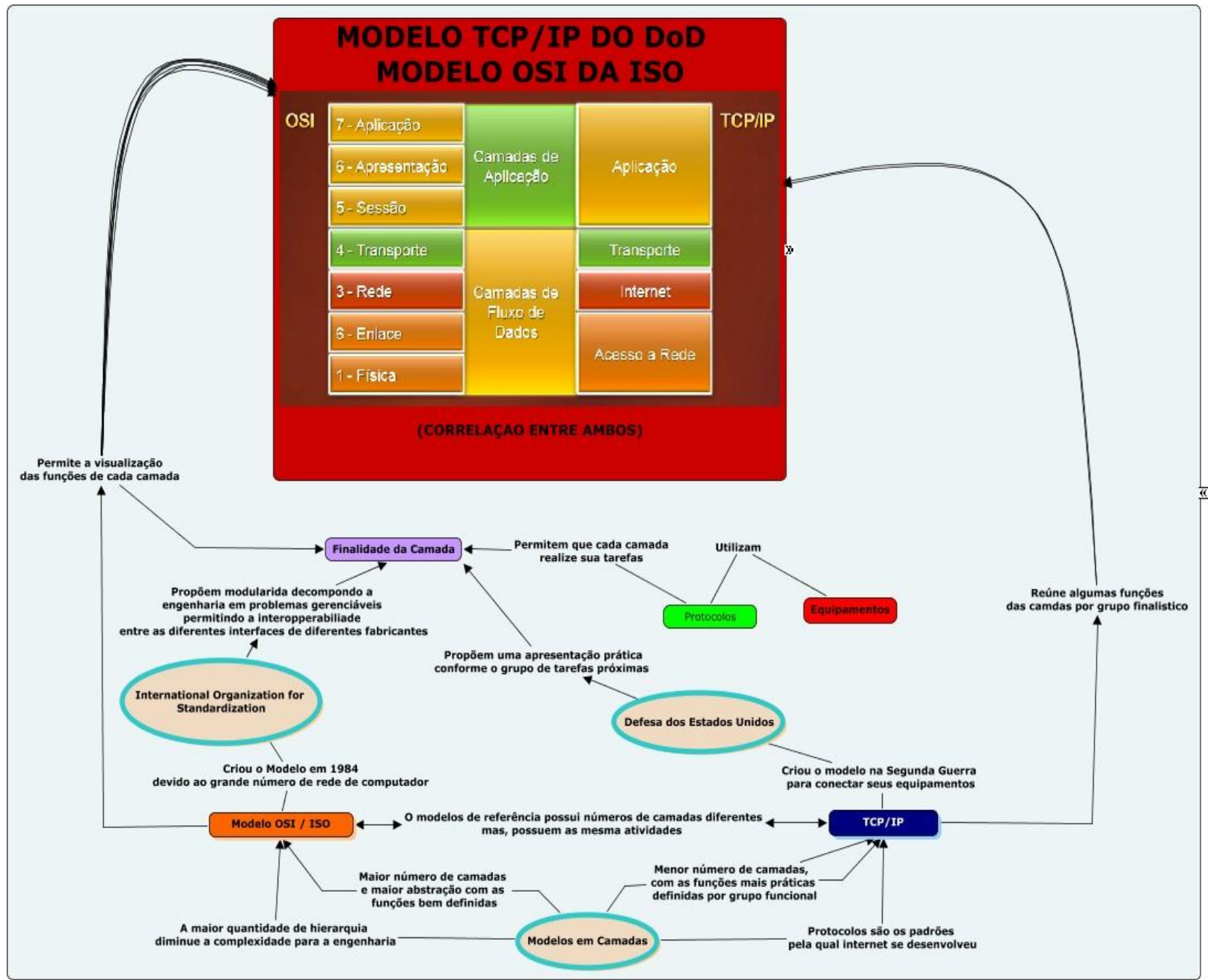

Figura 2 - Mapa conceitual inicial sobre "Comparação entre os modelos em camadas" 


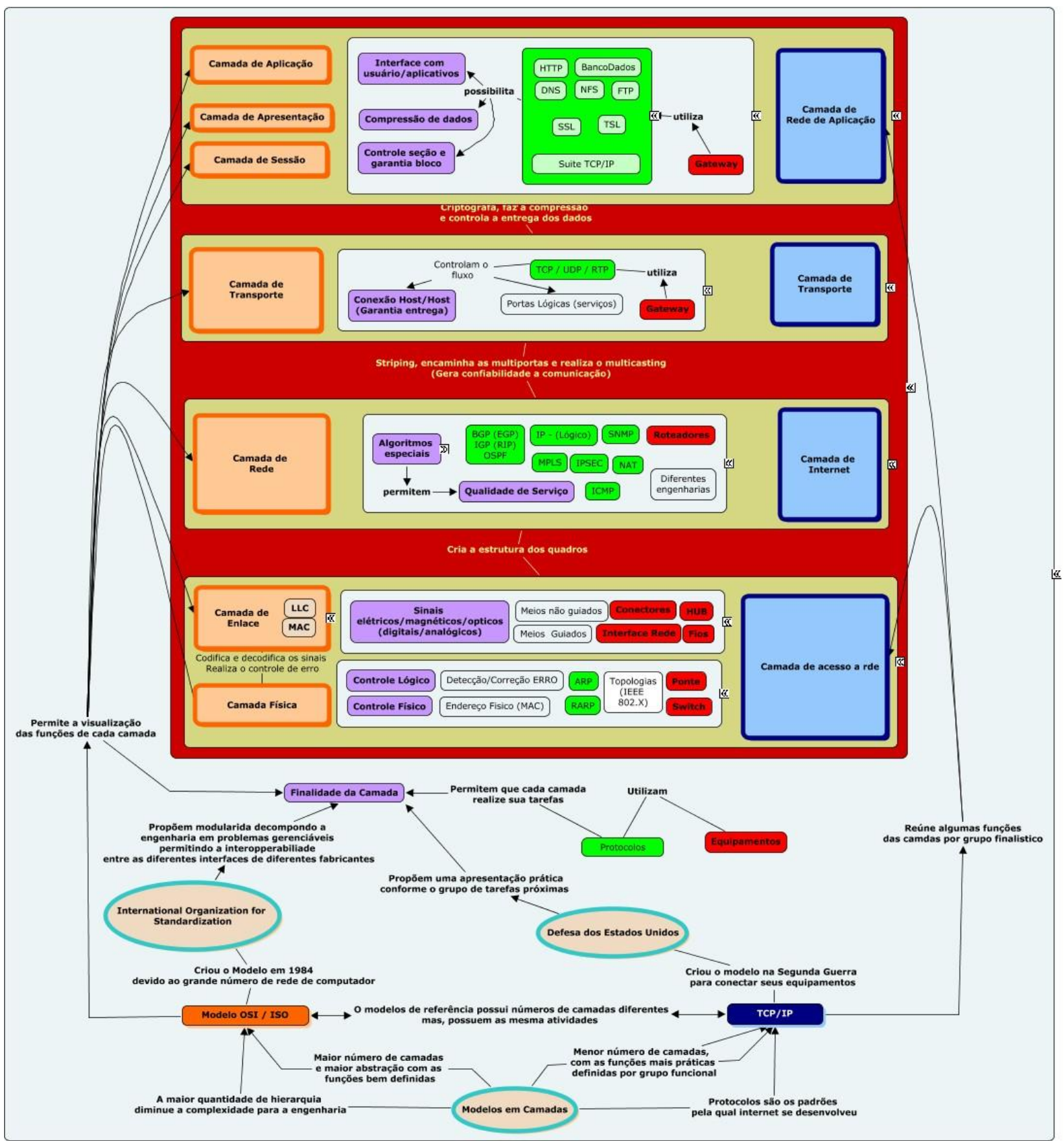

Figura 3 - Mapa conceitual final sobre "Comparação entre os modelos em camadas"

$\mathrm{Na}$ construção dos mapas é fundamental a observação constante do professor, pois, segundo Silveira, "o grande desafio para o professor é ajudar o aluno a utilizar, de forma consciente e produtiva, o seu potencial de pensamento" (Silveira, 2008, p. 86).

Ao final da realização do mapa foi pedido que os alunos explicassem o mesmo para todos os colegas, já que, segundo Moreira, "mapas conceituais devem ser explicados por quem os faz; ao explicá-lo a pessoa externaliza os significados" (Moreira, 2010, p. 15). Neste momento os alunos puderam apresentar todo conteúdo aprendido demonstrando que a utilização do mapa facilitou a aprendizagem. Nas palavras de Silveira, "os mapas conceituais são instrumentos diferentes que devem ser avaliados, sobretudo, qualitativamente, a fim de se obter as evidências de aprendizagem significativa" (Silveira, 2008, p.87).

Após as aulas ministradas sobre o modelo em camadas, foram observadas duas vertentes. A primeira foi que o aprendizado através do mapa conceitual ficou mais fácil para a visualização das diferenças e equidades. A segunda vertente despertou um grande interesse 
pelo aprendizado através de mapa conceitual, em que os educando pediram novos formatos para demais disciplinas. Sendo então disponibilizada pela coordenação do curso uma oficina sobre o assunto.

A referida oficina rendeu trabalhos extraclasse com o uso do CmapsTools, onde foi possível observar outras aplicabilidades com diversas cadeiras do presente curso como por exemplo, a disciplina empreendedorismo, sendo construído no modelo aranha.

É importante ressaltar que todos afirmaram que, durante a confecção do mapa conceitual conseguiram perceber quais eram as suas dificuldades em relação ao conteúdo estudado e que também acharam importante discutir com os colegas o assunto investigado durante a confecção do mapa.

Todos os alunos responderam que acharam fácil utilizar a ferramenta mapa conceitual e afirmaram também, que tiveram uma visão mais abrangente do assunto estudado em sala de aula.

\section{Análise e discussões}

Segundo opiniões colhidas em sala e observando a reação dos educando, estes de forma geral não tiveram dificuldades para utilizar a ferramenta de construção dos mapas conceituais, após a oficina. Conseguiram ter uma visão mais abrangente do conteúdo estudado. A construção em conjunto dos mapas conceituais possibilitou a interação entre os indivíduos envolvidos no processo ensino-aprendizado, trazendo também a possibilidade de inclusão e valorização do conhecimento tácito de cada estudante.

Outro aspecto observado foi à criatividade dos estudantes no momento de construção dos mapas. Os alunos se sentiram bastante motivados, tanto na hora da criação, quanto no momento de explanação de suas atividades extraclasse, possibilitando uma boa avaliação, por parte dos professores, de diversas áreas.

Para a visão do docente ficou a possibilidade de que novas interações com os mapas já confeccionados aumentam as possibilidades de se passar às informações de formas mais dinâmicas e menos estáticas.

\section{Conclusões}

$\mathrm{Na}$ visão do processo ensino-aprendizado é importante reconhecer os subsunçores préexistentes na estrutura cognitiva dos alunos para que se possa planejar da melhor forma a utilização dos mapas conceituais como recurso didático.

No ultimo período do presente curso foi utilizado o mapa conceitual no modelo estruturado na disciplina redes de computadores, com a aplicabilidade deste formato de aula foi detectado que os alunos se interagiam mais. Por isso o pedido de uma oficina para ensinar a utilização da ferramenta.

$\mathrm{Na}$ visão do indivíduo quanto à utilização do mapa conceitual para montar seu conhecimento, este se tornaram capazes de perceber as lacunas, seja, nos conceitos ou nas conexões entre os mesmos.

Cabe ao professor conhecer a ferramenta e elaborar um bom planejamento para que as atividades em sala de aula sejam desenvolvidas de forma eficiente.

Os mapas conceituais se apresentaram como um ótimo recurso de ensino-aprendizagem, além de apresentarem como uma ferramenta dinâmica para o processo ensino-aprendizado, permitindo a inserção de novos conceitos e conexões, caso seja preciso. 


\section{Referências Bibliográficas}

ALBUQUERQUE, R. C.; MANSUR, A. F. U.; BASTOS, H. P. P.; LEITE, M. L. T. T.; AMORIM, M. J. V.; MACEDO, S. H. Tecnologias da Informação e da comunicação no PROEJA: contribuições, possibilidades e desafios. Dialogando PROEJA - algumas contribuições, p. 91-106, 2009.

AUSUBEL, D.; NOVAK, J.; HANEISIAN, H. Psicologia Educacional. 2. ed. Rio de Janeiro: Interamericana, 1980.

BOLZANI, Caio Augustus Morais. Residências Inteligentes: Domótica; Redes Domésticas; Automação Residencial. 2004. Editora livraria da Física. São Paulo- 2004 - $1^{\circ}$ Ed.

CARVALHO, A. S.; BARONE, D. A. C.; ZARO, M. A. A aprendizagem significativa no ensino de engenharia de controle de automação. RENOTE. Revista Novas Tecnologias na Educação. V. 8 N 3, dezembro, 2010.

COLL, César. Aprendizagem escolar e construção do conhecimento. Porto Alegre: Artmed, 2000.

COSTA, José Wilson. Informática na educação - uma síntese. Campinas, SP: Papirus, 1998.

DUTRA, Ítalo Modesto; JOHANN, Stéfano Pupe. Por uma abordagem construtivista dos mapas conceituais. Disponível em: <http://mapasconceituais.cap.ufrgs.br/acai/ambiente/ atividades/ativ_33/10:08:54_03-08-2006_117_mapasconstrutivismo.pdf $>$ Acesso em: $10 \mathrm{de}$ outubro de 2011.

IFF, Instituto Federal Fluminense. Disponível em: 〈www.iff.edu.br> Acesso em: 10 de outubro de 2011.

LEITE, M. L. T. T.; BASTOS, H. P. P.; LIMA, J. V.; BIAZUS, M. C. V.; MANSUR, A. F. U.; AMORIM, M. J. V.; ALBUQUERQUE, R. C.; MACEDO, S. H. Hiperdocumento aplicado a alunos do PROEJA: relato de experiência. Revista Ciências \& Idéias N.1, vol. 2, set.2010.

LEITE, M. L. F. T. T.; FRANCO, S. R. K. PROEJA e Letramento Digital. Cadernos de Informática. Vol. $6 \mathrm{~N}^{\circ} 1.2011$.

MOREIRA, M. A. A teoria da Aprendizagem Significativa e sua implementação em sala de aula. Brasília: Editora Universidade de Brasilia, 2006.

Mapas Conceituais e Aprendizagem Significativa. 1997. Disponível em: $<$ http $/ /$ www.if.ufrgs.br/ moreira/mapasport.pdf> Acesso em 10 de outubro de 2011.

Mapas Conceituais e Aprendizagem Significativa São Paulo: Centauro, 2010. 
M. A., MASINI, E. F. S. Aprendizagem Significativa - A Teoria de David Ausubel. São Paulo: Centauro, 2001.

SILVEIRA, F. P. R. A., Levantamento preliminar de habilidades prévias: subsídios para a utilização de mapas conceituais como recurso didático. In: Revista Eletrônica Experiências em Ensino de Ciência, v. 3, p. 85-96, 2008.

TANENBAUM, Andrew S. Rede de computadores. Editora Campus, 2003. 